\title{
Prediction and extraction of microRNA2target interactions associated with leukemia
}

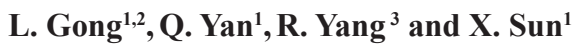 \\ ${ }^{1}$ State Key Laboratory of Bioelectronics, \\ School of Biological Science and Medical Engineering, \\ Southeast University, Nanjing, China \\ ${ }^{2}$ College of Computer, Nanjing University of Posts and Telecommunications, \\ Nanjing, China \\ ${ }^{3}$ Faculty of Computer Engineering, Huaiyin Institute of Technology, \\ Huai' an, China \\ Corresponding author: X. Sun \\ E-mail: xsun@seu.edu.cn
}

Genet. Mol. Res. 13 (1): 2120-2129 (2014)

Received November 30, 2012

Accepted April 10, 2013

Published March 24, 2014

DOI http://dx.doi.org/10.4238/2014.March.24.19

\begin{abstract}
MicroRNAs are small, non-coding RNAs that regulate gene expression by suppressing mRNA translation or inducing mRNA degradation, and have been implicated in a growing number of diseases. To understand microRNAs' function, it is vital to identify microRNA2target interactions. This work explores the prediction and extraction of leukemia-associated microRNA2target interactions, based on text mining. We extracted 371 interactions of microRNA2targets that, from prior knowledge, could be related to leukemia. By measuring similarities between unknown and known targets, the study could also predict some interactions of microRNA2targets. To analyze the prioritized data, the proposed approach identified some microRNA2target interactions, 17 of which were validated by other evidences. The remaining unconfirmed interactions provide a resource for leukemia researchers. Experimental results show the work has promise for predicting and extracting interactions of microRNA2targets
\end{abstract}


related to leukemia.

Key words: MicroRNA; Target genes; Interactions of microRNA2target; Leukemia

\section{INTRODUCTION}

Leukemia is a cancer that starts in the marrow and soft tissue inside most bones, and is characterized by an abnormal increase in immature blood cells (Joshi et al., 2000). It is increasingly being recognized as one of a spectrum of diseases, called hematological neoplasms, affecting the blood, bone marrow, and lymphoid system (Farnault et al., 2012). It is reported that 209,000 people have died from leukemia, with approximately $90 \%$ of leukemias being diagnosed in adults around the world in 2000 (Shibuya et al., 2002). Clinically and pathologically, leukemia can be subdivided into acute and chronic forms. Acute leukemia is characterized by a rapid increase in the number of immature blood cells, whereas chronic leukemia worsens slowly, causing symptoms for years. Leukemia is a debilitating and deadly disease that gets widespread attention. With the development of molecular biology technology, research on leukemia now ranges from group medicine to cell and molecular biology. Although many factors are considered related to leukemia, the exact etiology remains unclear. Current studies show that genetic factors also contribute to the development of leukemia. Mahjoubi and Akbari (2012) found that over-expression of the MRP1 gene occurred in most Iranian pediatric leukemia patients at relapse. Yoshimi and Kurokawa (2011) reported histone methyl-transferase and demethylase play key roles in leukemogenesis. It is reported that TET2 defects are present in hematopoietic stem cells, and TET2 mutations may be with a heterozygous deletion in leukemia patients (Delhommeau et al., 2009). Calin et al. (2002) describe the regulation of microRNA genes in chronic lymphocytic leukemia. MicroRNAs and regulatory targets are potentially druggable in leukemia. Given the high cost and laborious nature of experimental validation, it is imperative that computational approaches are developed for exploring both microRNA and the target genes involved in leukemia. Arjas and Liu (1996) proposed a non-parametric, multiplicative hazard regression model in their research on leukemia. Fox et al. (2006), in their work on leukemia, developed PROforma, a formal language for modeling clinical processes along with associated tools for creating decision support. Zhou et al. (2006) proposed multi-class cancer classification, using multinomial probit regression with Bayesian gene selection to analyze acute leukemia data. With the rapid increase in publication of valuable bioinformation, it becomes possible to mine leukemia-associated biomedical data from the literature. Generally, predictive computational approaches for identification of microRNA2 target interactions look for target sites based on thermodynamics, binding structure and conservation (Lewis et al., 2003; Lai, 2004). Text mining has the potential of discovering biomedical knowledge in cost-effective manner (Gong et al., 2012). In this paper, we propose a text-mining approach to predict and obtain interactions of microRNA2target genes related to leukemia. The approach first identifies biomedical entities from the literature that are related to leukemia that contain microRNAs and protein-coding genes. Using prior knowledge (mature database of microRNA2target interactions), the approach correctly identified interactions of microRNA2targets, and predicted interactions by using other bioinformatic tools. To the best of our knowledge, this is the first time microRNA2target interactions related to leukemia have been found by text mining. Experimental results show the approach is promising for acquiring microRNA2target interactions, and represents a new way of delving into this area of leukemia research. 


\section{MATERIAL AND METHODS}

\section{Architecture of our methods}

Genes are the molecular units of heredity of a living organism, holding the information to build an organism's cells and pass genetic traits to offspring. MicroRNAs, also called miRNAs, are small, non-coding ribonucleic acid molecules found in eukaryotic cells. They are approximately 22 nucleotides in length and are involved in critical biological processes, such as translational regulation, cell apoptosis, and neoplasm metastasis. Previous research reported that about $1 \%$ of all human genes are microRNA genes that regulate more than $10 \%$ of protein production (John et al., 2004). MicroRNAs are regarded as a critical factor in targeting oncogenes in tumorigenesis (Chu et al., 2012). MicroRNAs and protein-coding genes are two key genetic factors in research on leukemia pathogenesis. In this research, we worked with material from the literature that is likely to be related to leukemia. We first obtained microRNA and protein-coding genes by text mining. We then applied bioinformatic analysis to that material to identify microRNA2target interactions. The framework of our approach for exploring those interactions is shown in Figure 1.

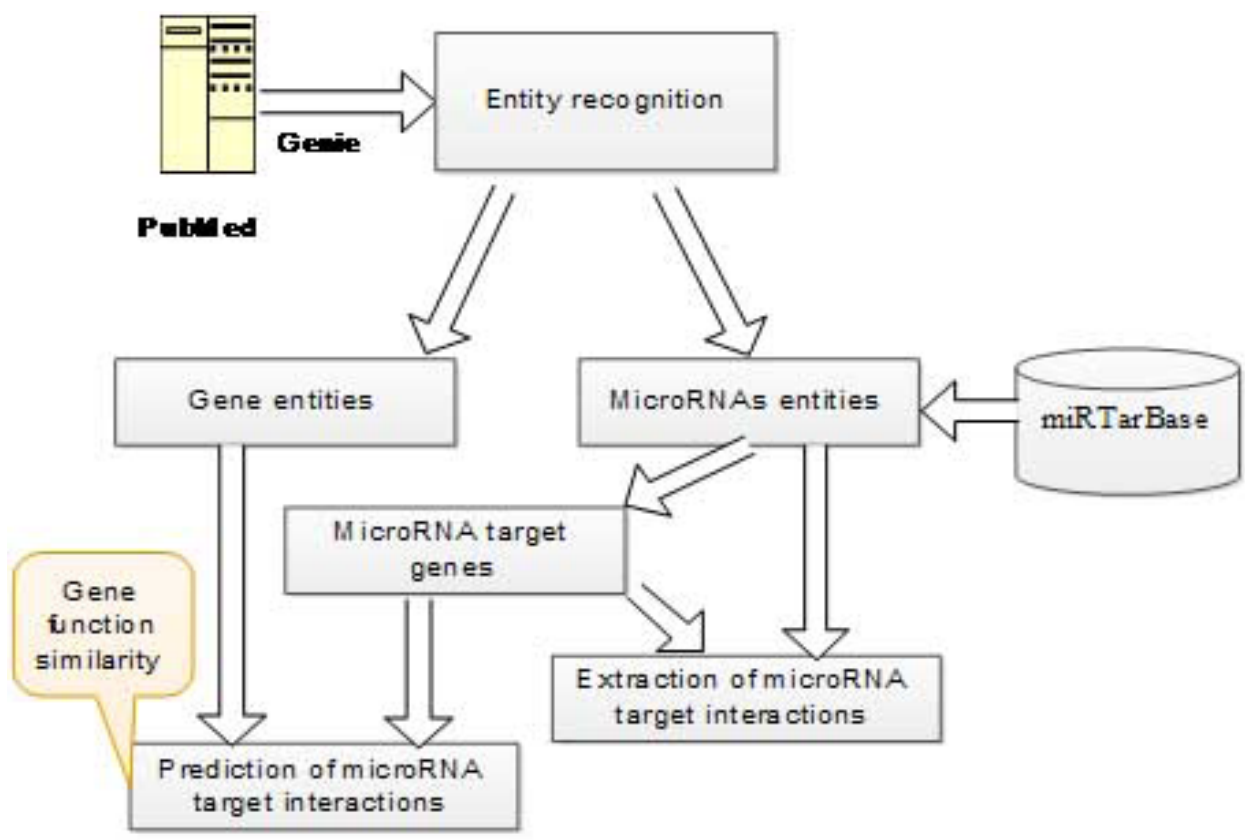

Figure 1. Pipeline of exploring microRNA2target interactions.

In Figure 1, the approach first recognizes microRNA and target gene entities in leukemia literature using Genie (Fontaine et al., 2011), and retrieves microRNAs and genes associated with the disease. It then finds the microRNA target genes and their interactions through miRTarBase (Hsu et al., 2011) and goes on to measure the similarities between known microRNA target genes and unknown functional genes to predict and prioritize microRNA target interactions. 


\section{Entity recognition}

Text mining tools could extract key pieces of information from the volume of literature in biomedical research (Gong et al., 2011; 2013). Genie, which is reportedly capable of up to $100 \%$ precision, is used to mine protein-coding genes and microRNAs from the leukemia literature. We obtained 305 protein-coding genes and 102 microRNAs using the keyword "leukemia" and searching the whole Medline database with Genie on May 9, 2012. Mined microRNAs and genes associated with leukemia are shown in Table 1 with the corresponding parameter settings.

Table 1. Mined microRNAs and genes associated with leukaemia.

\begin{tabular}{lccc}
\hline Categories & P value & False discovery rates (FDR) & Numbers \\
\hline Protein-coding genes & $<0.01$ & $<$ E-10 & 305 \\
MicroRNAs & $<0.05$ & $<0.05$ & 102 \\
\hline
\end{tabular}

\section{Extraction of microRNA2target interactions}

MicroRNA2target interactions (MTIs) are extracted from the literature associated with leukemia with miRTarBase, a database of validated microRNA-target interactions that we confirmed accumulates a larger and more frequently updated collection of MTIs than other similar resources. In the study, miRTarBase acts as the data source for mapping MTIs related to the leukemia literature. By mapping between the extracted microRNAs and the miRTarBase database, we found 135 target genes and 371 interactions of microRNA2target, as shown in Supplementary Table S1. The visual network of microRNA-target interactions was developed by pajek (Milenković et al., 2008) with functional semantics. It is shown in Figure 2.

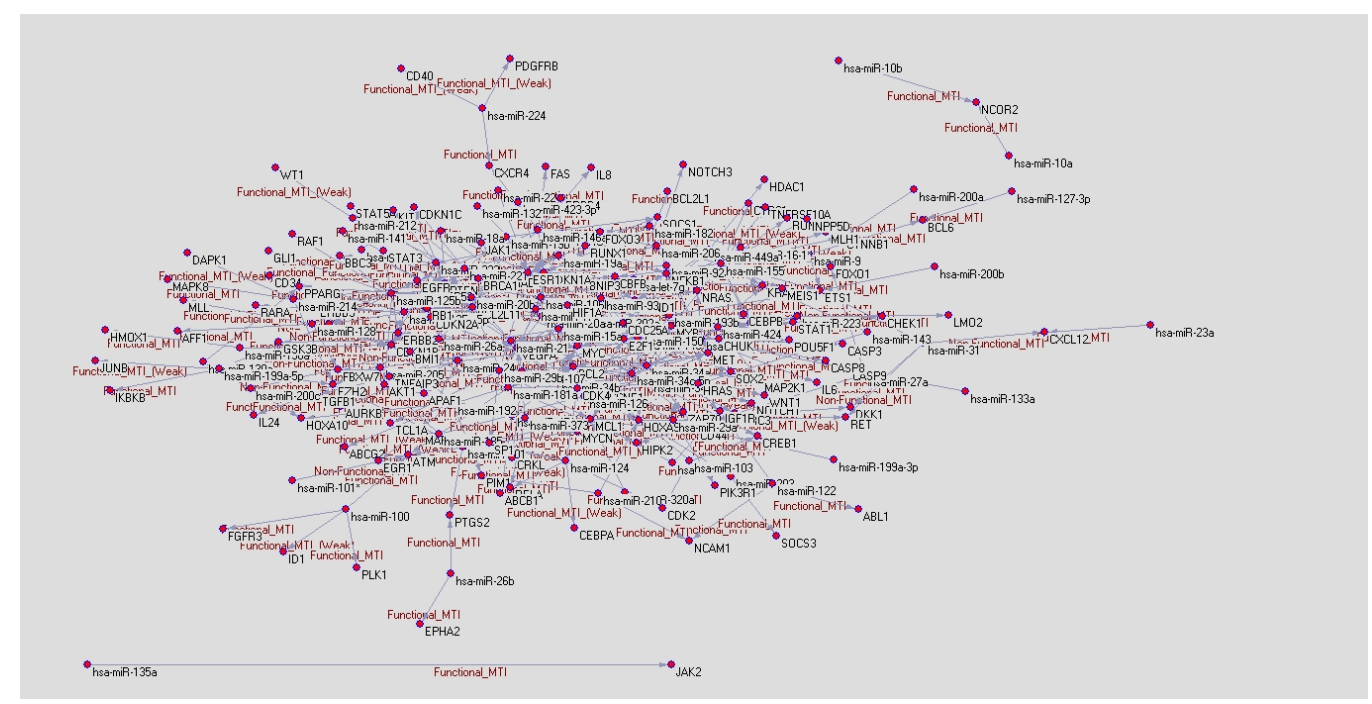

Figure 2. Visual network of microRNA2target interactions. 
In Figure 2, red vertices represent biomedical entities, and the edges represent the regulated relations between vertices. The labels on the edges describe the strength of interactions by annotation with Function_MTI or Function_MTI(Weak). For example, annotation of the interaction between has-miR-135a and JAK2 is Function_MTI, and the annotation between hasmiR-124 and CEBPA is Function_MTI(Weak). The visual network of microRNA2target interactions with its functional semantic annotations clearly presents the strength of the interactions.

\section{Prioritized unknown genes}

In Table S1, 135 of 305 protein-coding genes act as the microRNA target genes of the MTIs validated by experiment. These known target genes obtained from the mapping are also correspondingly related to leukemia. The remaining 170 protein-coding genes, which are not known microRNA target genes, are referred to as unknown genes. The unknown genes are predicted according to similarity measurements that are also applied in work with the aid of known microRNA target genes (Xiao et al., 2012). To clearly elaborate the predicted results, we pre-prioritize the unknown genes by Endeavour (Tranchevent et al., 2008), a bioinformatic tool for gene prioritization that is used to measure the interaction similarities between unknown and known target genes. In Endeavour, the known target genes are used as the training genes, and the unknown genes as the test genes. Figure 3 shows the top 20 genes predicted by Endeavour along with the interactions' data source.

\begin{tabular}{|c|c|c|c|c|c|c|c|}
\hline Global & Bind & BioGrid & Hprd & InNetDb & Intact & Mint & String \\
\hline CFLAR & APEX1 & BRAF & CFLAR & CEBPD & TNFRSF8 & & $T A$ \\
\hline STATSB & EGF & CFLAR & STATEB & NFE2L2 & JUN & Binx & CSF3R \\
\hline BRAF & CHEK2 & KLF6 & IRF8 & TNFRSF8 & BCL2AI & EGF & BIRC7 \\
\hline BAD & BRAF & TP73 & CEBPD & MAX & BAD & MAX & EPAS1 \\
\hline JUN & PIKЗСG & A & \begin{tabular}{|l|l|} 
TNFRSF \\
\end{tabular} & CFLAR & NBN & GAB2 & NFKB2 \\
\hline BIRC7 & HGF & IRF8 & BIRC7 & FLT4 & PTK2B & $5 \mathrm{KP2}$ & ERG \\
\hline BIRC5 & GATA1 & $\mathrm{BCR}$ & MS4AA1 & JUN & $B A X$ & INSL3 JJAKK3 & MALT1 \\
\hline $\mathrm{BCL2A1}$ & RAD51 & CEBPD & TWIST1 & SRC & FASLG & CFLAR & $\begin{array}{c}\text { TNFSF12 ITN } \\
\text { FSF13 }\end{array}$ \\
\hline EGF & $\begin{array}{l}\text { CFLAR } \\
\text { CFLe }\end{array}$ & TNFRSF10B & BIRC5 & PIK3CA & BRAF & KDR & FLT3 \\
\hline KLF6 & $P A \times 5$ & GSTP1 & BCl.2A1 & KLF6 & PIK3CG & PRKCD & FCER2 \\
\hline NFKB2 & AURKA & BIRC7 & FLT1 & BCL2A1 & EPHB4 & FOXNon 1 & ANGPT2 \\
\hline CEBPD & KLF6 & MS4AA1 & ZMMYMU2 & SFRP1 & Binx & TERT & BAÁLC \\
\hline $5 \mathrm{KP2}$ & CSF1R & TWIIST1 & NBN & AURKA & FIP1L1 & $5 Y K$ & $T L \times 3$ \\
\hline MAX & MAPK1 & STK11 & Foxom 1 & LYN & AKT2 & CREBBP & NANOG \\
\hline GAB2 & ZBTB16 & CSF1R & $T L \times 3$ & BAD & HGF & STAT5B & $\mathrm{BIRC} 5$ \\
\hline CHEK2 & FLT1 & NFKB2 & CD19 & 5KP2 & PTK2 & GATA1 & IRF8 \\
\hline FLT1 & RUN\3 & BIRC5 & BRAF & CDKN2B & EGF & RAD51 & TNFSF10 \\
\hline mDim 2 & FLT3 & CSF3R & FGFR4 & LCK & CBL & TP73 & \begin{tabular}{|l|l|} 
TNFRSF13C \\
\end{tabular} \\
\hline TNFRSF10B & BCL2A1 & INSL3 JJAK3 & CSF1R & RASSF1 & SHC1 & CSF $3 R$ & FGFR4 \\
\hline MAPK1 & $\mathrm{AXL}$ & PDGFRA & TP73 & IKZF1 & $M A X$ & NFKBIÁ & PIK3CA \\
\hline
\end{tabular}

Figure 3. Top twenty prioritized results of unknown genes. 
In Figure 3, Endeavour uses Q statistics (Stuart et al., 2003) that are calculated from all rank ratios using the joint cumulative distribution of an $\mathrm{N}$-dimensional order statistic to rank the test genes, as shown in Figure 4. These genes are represented as boxes, and the top ranking 16 genes are given colors in order to easily find the rank of a given gene obtained for each model. The predicted genes with global rank of the top 16 are attributed a random background color (e.g. "BRAF" in "Global" column). The predicted genes with global rank $>16$ have a white background color (e.g., "FLT4" in "InNetDb" column). Predicted genes in red achieve a maximum dissimilarity score (most dissimilar to the training genes, e.g., "PIK3CG" in "Bind" column).

\begin{tabular}{|c|c|c|c|c|c|c|c|c|c|c|c|c|c|c|c|c|}
\hline \multirow{2}{*}{ Gene } & \multicolumn{2}{|c|}{ Global } & \multicolumn{2}{|c|}{ Bind } & \multicolumn{2}{|c|}{ BioGrid } & \multicolumn{2}{|c|}{ Hprd } & \multicolumn{2}{|c|}{ InNetDb } & \multicolumn{2}{|c|}{ Intact } & \multicolumn{2}{|c|}{ Mint } & \multicolumn{2}{|c|}{ String } \\
\hline & rank & score & rank & score & rank & score & rank & score & rank & score & rank & score & rank & score & rank & score \\
\hline CFLAR & 1 & 0.00094 & 9 & 1 & 2 & 1 & 1 & 1 & 5 & 1.03 & 62 & 2.14 & 8 & 1 & 56 & 1.02 \\
\hline STAT5B & 2 & 0.00356 & 60 & 2 & 5 & 1 & 2 & 1 & 28 & 1.13 & 35 & 1.5 & 15 & 1.2 & 1 & 1 \\
\hline BRAF & 3 & 0.0047 & 4 & 1 & 1 & 1 & 17 & 1.06 & 66 & 1.32 & 9 & 1 & 30 & 1.5 & 26 & 1.01 \\
\hline BAD & 4 & 0.00608 & 23 & 1.2 & 45 & 1.31 & 35 & 1.17 & 15 & 1.07 & 4 & 1 & 1 & 1 & 42 & 1.02 \\
\hline JUN & 5 & 0.00916 & 27 & 1.3 & 32 & 1.21 & 33 & 1.16 & 7 & 1.05 & 2 & 1 & 28 & 1.44 & 63 & 1.03 \\
\hline BIRC7 & 6 & 0.0143 & 146 & none & 11 & 1 & 6 & 1 & 124 & 2.33 & 21 & 1.25 & 22 & 1.33 & 3 & 1 \\
\hline BIRC5 & 7 & 0.0143 & 57 & 2 & 17 & 1.11 & 9 & 1 & 46 & 1.21 & 37 & 1.5 & 40 & 1.6 & 15 & 1 \\
\hline BCL2A1 & 8 & 0.0169 & 19 & 1.17 & 21 & 1.13 & 10 & 1 & 11 & 1.06 & 3 & 1 & 78 & none & 109 & 1.06 \\
\hline EGF & 9 & 0.0262 & 2 & 1 & 34 & 1.25 & 22 & 1.09 & 129 & 2.68 & 17 & 1 & 3 & 1 & 103 & 1.05 \\
\hline KLF6 & 10 & 0.0347 & 12 & 1 & 3 & 1 & 99 & 1.5 & 10 & 1.05 & 63 & 2.2 & 46 & 1.71 & 74 & 1.03 \\
\hline NFKB2 & 11 & 0.0362 & 48 & 2 & 16 & 1.09 & 64 & 1.29 & 38 & 1.17 & 49 & 1.9 & 62 & 2.04 & 5 & 1 \\
\hline CEBPD & 12 & 0.0362 & 93 & none & 8 & 1 & 4 & 1 & 1 & 1 & 84 & none & 101 & none & 54 & 1.02 \\
\hline SKP2 & 13 & 0.0375 & 53 & 2 & 55 & 1.36 & 26 & 1.13 & 16 & 1.08 & 24 & 1.29 & 6 & 1 & 94 & 1.04 \\
\hline $\operatorname{MAX}$ & 14 & 0.0476 & 59 & 2 & 93 & 2 & 107 & 1.62 & 4 & 1 & 20 & 1.18 & 4 & 1 & 108 & 1.05 \\
\hline GAB2 & 15 & 0.0511 & 159 & none & 28 & 1.17 & 23 & 1.1 & 150 & none & 22 & 1.25 & 5 & 1 & 23 & 1.01 \\
\hline CHEK2 & 16 & 0.0525 & 3 & 1 & 75 & 1.57 & 71 & 1.31 & 36 & 1.16 & 27 & 1.33 & 31 & 1.5 & 45 & 1.02 \\
\hline FLT1 & 17 & 0.0535 & 16 & 1 & 127 & 7 & 11 & 1 & 57 & 1.26 & 38 & 1.5 & 51 & 2 & 22 & 1.01 \\
\hline MDM2 & 18 & 0.0564 & 46 & 2 & 48 & 1.33 & 61 & 1.26 & 65 & 1.32 & 46 & 1.69 & 23 & 1.33 & 27 & 1.01 \\
\hline $\begin{array}{l}\text { TNFRSF } \\
\text { 10B }\end{array}$ & 19 & 0.0584 & 94 & none & 9 & 1 & 5 & 1 & 30 & 1.14 & 50 & 2 & 67 & 3 & 35 & 1.01 \\
\hline MAPK1 & 20 & 0.0638 & 14 & 1 & 60 & 1.39 & 67 & 1.3 & 39 & 1.17 & 70 & 3 & 43 & 1.6 & 55 & 1.02 \\
\hline
\end{tabular}

Figure 4. Scores of the prioritized top twenty genes.

After prioritization with Endeavour, we consider the top 20 genes as the most likely to be microRNA target genes. Therefore, we will focus on searching MTIs from the top 20 genes in the next section.

\section{Prediction of microRNAs2target interactions}

Considering the interactions of microRNA2target, the top 20 prioritized genes are used in this section to further refine the predictions. The top 20 genes together with the 135 known target genes are subjected to microRNA target enrichment analysis using WebGestalt (Zhang et al., 2005). Of the 20 genes, four, FLT1, MDM2, GAB2, and STAT5B, are target genes. The interactions of microRNA2target related to the four genes are shown in Table 2.

Following the work of Xiao et al., 2012, gene functions are also used to predict microRNA targets. Thus, we hypothesize that if an unknown gene has similar functions to the known target gene, then the unknown gene could be a target of MTIs related to the known target gene. We use the DAVID tool (Sherman et al., 2007) to analyze gene functional clas- 
sifications. Of the top 20, seven are in five gene groups, showing that these genes have similar functions in the whole genome, as shown in Table 3. The greater enrichment scores of a group show the genes in it to be more relevant than are those of other groups.

\begin{tabular}{lll}
\multicolumn{2}{c}{ Table 2. Nine predicted interactions obtained from microRNA target enrichment analysis. } \\
\hline No. & mircroRNA & Target gene \\
\hline 1 & hsa-mir-19A & STAT5B (signal transducer and activator of transcription 5B) \\
2 & hsa-mir-19B & \\
3 & hsa-mir-377 & MDM2 (Mdm2 p53 binding protein homolog) \\
4 & hsa-mir-218 & GAB2 (GRB2-associated binding protein 2) \\
5 & hsa-mir-20A & FLT1 (fms-related tyrosine kinase 1) \\
6 & hsa-mir-20B & \\
7 & hsa-mir-519D & \\
8 & hsa-mir-17-5P & \\
9 & hsa-mir-106A & \\
\hline
\end{tabular}

\begin{tabular}{|c|c|c|}
\hline Group & ENTREZ_GENE_ID & Score \\
\hline 1 & $\begin{array}{l}865,1385,6774,2309,4299,1050,6657,2308,9612,7390,6688,6667,3726,861,2735,3065,1869,860, \\
1316 \text { (KLF), 1051, 1958, } 203\end{array}$ & 20.96 \\
\hline 2 & $\begin{array}{l}28996,5894,5604,1111,1147,11200 \text { (CHEK2), 2932, 5292, 9212, 5599, 1432, 3551, 5594(MAPK1), } \\
1017,3265,472,673 \text { (BRAF), 1019, 1612, 25, 5347 }\end{array}$ & 16.45 \\
\hline 3 & $3205,5460,4602,1052$ (CEBPD), 3206, 4211 & 12.93 \\
\hline 4 & 841, 8837(CFLAR), 836, 842 & 10.59 \\
\hline 5 & 355,8795 (TNFRSF10B), 8797, 27113 & 10.1 \\
\hline
\end{tabular}

Table 3 shows the seven genes predicted to be microRNA target genes in terms of functional similarities. For example, gene TNFRSF10B (Gene_ID: 8795) is in the fifth group that contains three known target genes: FAS (Gene_ID: 355), TNFRS10A (Gene ID:8797), and $B B C 3$ (Gene_ID:27113), as shown in Figure 5. Enrichment analysis shows the functions of gene TNFRSF10B involved apoptosis, cell death, and regulation, which potentially could produce leukemia. It is from the TNF-receptor superfamily whose members contain an intracellular death domain. It could also be activated by tumor necrosis factor-related apoptosis, and it transduces an apoptosis signal. TNFRSF10B was predicted to be an MTI target gene (Table 4) by the three known target genes from the 371 MTIs (Table S1) in same group.

More details of the predicted interactions related to the seven genes are shown in Table S2. There are five interactions confirmed by miRDB (Wang, 2008), which is a microRNA target prediction and functional annotation database that uses a wiki model, as shown in Table 5.

Analyzing the prioritized top 20 genes, we obtained 17 interactions with MTIs that involve seven genes: FLT1, MDM2, GAB2, STAT5B, TNFRSF10B, MAPK1, and BRAF. This was validated by other bioinformatic tools. The expression of FLT1 is found in 12 of 13 (93.3\%) acute myeloid leukemia (AML) cell lines, and 53.1\% of 31 AML patient's bone marrow mononuclear cells expressed FLT1 mRNA (Wang et al., 2003). MDM2 was identified as a tumor-associated antigen in chronic lymphocytic leukemia (Mayr et al., 2006). GAB2 is essential for the major signal transduction pathway, and regulates a number of key cellular processes. It also acts as the driver of chronic myeloid leukemia (Wöhrle et al., 2013). Nuclear accumulation of STAT5A as a result of activation by FLT3-ITD is an oncogene found in acute 
myeloid leukemia (Chatain et al., 2012). TNFRSF10B-induced apoptosis in chronic lymphocytic leukemia cells is one of the most promising candidates for cancer therapeutics (Natoni et al., 2007; Secchiero et al., 2009). MAPK1 is involved in a wide variety of cellular processes, such as proliferation, differentiation, transcription regulation and development, correlated with acute myelogenous leukemia (Vey et al., 2004). Mutation of the $B R A F$ gene is associated with leukemia, as reported in a study by Ewalt et al., 2012. From this analysis, most of the predicted seven target genes are related to leukemia. Therefore, the predicted MTIs could play a critical role in leukemia research. The remaining interactions in Table S2 are novel predictions that could be useful to biomedical researchers in the leukemia area.

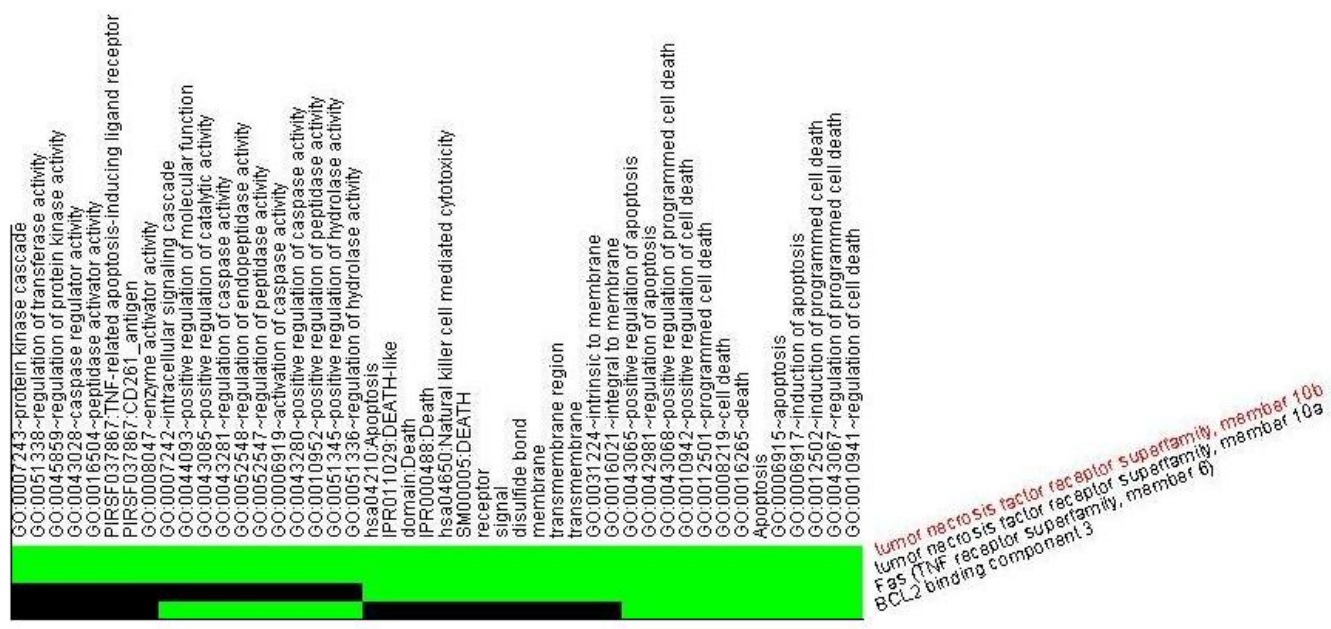

Figure 5. 2D-view of functional cluster of the fifth group. Green box $=$ corresponding terms association positively reported. Black box $=$ corresponding terms not reported yet.

Table 4. Predicted three MTIs related to gene TNFRSF10B.

\begin{tabular}{llll}
\hline MircroRNA & Target gene & Type & Predicted target gene \\
\hline hsa-miR-146a & FAS & Functional MTI & TNFRSF10B \\
hsa-miR-155 & 355 & & \\
& TNFRSF10A & Functional MTI (Weak) & \\
hsa-miR-221 & 8797 & Functional MTI & \\
& BBC3 & & \\
\hline
\end{tabular}

Table 5. Five interactions of microRNA2target using miRDB.

\begin{tabular}{llll}
\hline NO. & MicroRNA & Target gene & Description \\
\hline 1 & hsa-miR-181a & MAPK1 & mitogen-activated protein kinase 1 \\
2 & hsa-miR-192 & BRAF & v-raf murine sarcoma viral oncogene homolog B1 \\
3 & hsa-miR-26a & MARK1 & mitogen-activated protein kinase 1 \\
4 & hsa-miR-27a & MARK1 & mitogen-activated protein kinase 1 \\
5 & hsa-miR-424 & MARK1 & mitogen-activated protein kinase 1 \\
\hline
\end{tabular}




\section{RESULTS}

The current paper presents an approach for prediction and extraction of microRNA2 target interactions related to leukemia that is based on text mining. With this approach, we obtained 102 microRNAs and 305 protein-coding genes related to leukemia from the biomedical literature. Based on the 102 microRNAs, 371 MicroRNAs target interactions were extracted and presented with functional semantics in a visual network containing 135 protein-coding genes from an authoritative MTI database. The predictions of MTIs were done by similarity measurements between unknown and known target genes. Endeavour was used to prioritize the unknown genes. The top 20 genes were considered the most likely to be microRNA target genes. Using bioinformatic tools, we obtained 17 MTIs that were validated by other, independent lines of evidence. The remaining unsupported interactions could act as experimental guides in leukemia studies.

Our approach is promising for extracting and predicting microRNA2target interactions related to leukemia.

\section{ACKNOWLEDGMENTS}

Research supported by the National Natural Science Foundation of China (\#60971099), the Natural Science Foundation of Jiangsu Province in China (\#BK20130417), and the Natural Science Foundation of the Higher Education Institutions of Jiangsu Province (\#12KJB520002).

\section{Supplementary Material}

\section{REFERENCES}

Arjas E and Liu L (1996). Non-parametric Bayesian approach to hazard regression: a case study with a large number of missing covariate values. Stat. Med. 15: 1757-1770.

Delhommeau F, Fontenay M and Vainchenker W (2009). Mutations in TET2 in myeloid cancers. Med. Sci. 25: 785-788.

Calin GA, Dumitru CD, Shimizu M, Bichi R, et al. (2002). Frequent deletions and down-regulation of micro- RNA genes miR15 and miR16 at 13q14 in chronic lymphocytic leukemia. Proc. Natl. Acad. Sci. U. S. A. 99: 15524-15529.

Chatain N, Ziegler P, Fahrenkamp D, Jost E, et al. (2012). Src family kinases mediate cytoplasmic retention of activated STAT5 in BCR-ABL-positive cells. Oncogene.

Chu YH, Tzeng SL, Lin CW, Chien MH, et al. (2012). Impacts of microRNA gene polymorphisms on the susceptibility of environmental factors leading to carcinogenesis in oral cancer. PLoS One 7: e39777.

Ewalt M, Nandula S, Phillips A, Alobeid B, et al. (2012). Real-time PCR-based analysis of BRAF V600E mutation in low and intermediate grade lymphomas confirms frequent occurrence in hairy cell leukaemia. Hematol. Oncol. 30 : 190-193.

Farnault L, Sanchez C, Baier C, Le TT, et al. (2012). Hematological malignancies escape from NK cell innate immune surveillance: mechanisms and therapeutic implications. Clin. Dev. Immunol. 2012: 421702.

Fontaine JF, Priller F, Barbosa-Silva A and Andrade-Navarro MA (2011). Genie: literature-based gene prioritization at multi genomic scale. Nucleic Acids Res. 39: W455-W461.

Fox J, Patkar V and Thomson R (2006). Decision support for health care: the PROforma evidence base. Inform. Prim. Care 14: 49-54.

Gong LJ, Sun X, Jiang DK and Gong ST (2011). AutMiner: a system for extracting ASD-related genes using text mining. J. Biol. Syst. 19: 113-125.

Gong LJ, Yang RG and Sun X (2013). BRES: Extracting multiclass biomedical relations with semantic network. Biomed. Eng. Appl. Basis. Commun. 25: 1. 
Gong L, Yan Y, Xie J, Liu H, et al. (2012). Prediction of autism susceptibility genes based on association rules. $J$. Neurosci. Res. 90: 1119-1125.

Hsu SD, Lin FM, Wu WY, Liang C, et al. (2011). miRTarBase: a database curates experimentally validated microRNAtarget interactions. Nucleic Acids Res. 39: D163-D169.

John B, Enright AJ, Aravin A, Tuschl T, et al. (2004). Human MicroRNA targets. PLoS Biol. 2: e363.

Joshi SS, Tarantolo SR, Kuszynski CA and Kessinger A (2000). Antitumor therapeutic potential of activated human umbilical cord blood cells against leukemia and breast cancer. Clin. Cancer Res. 6: 4351-4358.

Lai EC (2004). Predicting and validating microRNA targets. Genome Biol. 5: 115.

Lewis BP, Shih IH, Jones-Rhoades MW, Bartel DP, et al. (2003). Prediction of mammalian microRNA targets. Cell 115: 787-798.

Mahjoubi F and Akbari S (2012). Multidrug resistance-associated protein 1 predicts relapse in Iranian childhood acute lymphoblastic leukemia. Asian Pac. J. Cancer Prev. 13: 2285-2289.

Mayr C, Bund D, Schlee M, Bamberger M, et al. (2006). MDM2 is recognized as a tumor-associated antigen in chronic lymphocytic leukemia by CD8+ autologous T lymphocytes. Exp. Hematol. 34: 44-53.

Milenković T, Lai J and Przulj N (2008). GraphCrunch: a tool for large network analyses. BMC Bioinformatics 9: 70.

Natoni A, MacFarlane M, Inoue S, Walewska R, et al. (2007). TRAIL signals to apoptosis in chronic lymphocytic leukaemia cells primarily through TRAIL-R1 whereas cross-linked agonistic TRAIL-R2 antibodies facilitate signalling via TRAIL-R2. Br. J. Haematol. 139: 568-577.

Secchiero P, Sblattero D, Chiaruttini C, Melloni E, et al. (2009). Selection and characterization of a novel agonistic human recombinant anti-TRAIL-R2 minibody with anti-leukemic activity. Int. J. Immunopathol. Pharmacol. 22: 73-83.

Sherman BT, Huang dW, Tan Q, Guo Y, et al. (2007). DAVID Knowledgebase: a gene-centered database integrating heterogeneous gene annotation resources to facilitate high-throughput gene functional analysis. BMC Bioinformatics 8: 426 .

Shibuya K, Mathers CD, Boschi-Pinto C, Lopez AD, et al. (2002). Global and regional estimates of cancer mortality and incidence by site: II. Results for the global burden of disease 2000. BMC Cancer 2: 37.

Stuart JM, Segal E, Koller D and Kim SK (2003). A gene-coexpression network for global discovery of conserved genetic modules. Science 302: 249-255.

Tranchevent LC, Barriot R, Yu S, Van VS, et al. (2008). ENDEAVOUR update: a web resource for gene prioritization in multiple species. Nucleic Acids Res. 36: W377-W384.

Vey N, Mozziconacci MJ, Groulet-Martinec A, Debono S, et al. (2004). Identification of new classes among acute myelogenous leukaemias with normal karyotype using gene expression profiling. Oncogene 23: 9381-9391.

Wang X (2008). miRDB: a microRNA target prediction and functional annotation database with a wiki interface. $R N A$ 14: 1012-1017.

Wang Y, Xiao ZJ, Liu P, Yang C, et al. (2003). Expression of vascular endothelial growth factor and its receptors KDR and Flt1 in acute myeloid leukemia. Zhonghua Xue Ye Xue Za Zhi. 24: 249-252.

Wöhrle FU, Halbach S, Aumann K, Schwemmers S, et al. (2013). Gab2 signaling in chronic myeloid leukemia cells confers resistance to multiple Bcr-Abl inhibitors. Leukemia 27: 118-129.

Xiao Y, Guan J, Ping Y, Xu C, et al. (2012). Prioritizing cancer-related key miRNA-target interactions by integrative genomics. Nucleic Acids Res. 40: 7653-7665.

Yoshimi A and Kurokawa M (2011). Key roles of histone methyltransferase and demethylase in leukemogenesis. J. Cell Biochem. 112: 415-424.

Zhang B, Kirov S and Snoddy J (2005). WebGestalt: an integrated system for exploring gene sets in various biological contexts. Nucleic Acids Res. 33: W741-W748.

Zhou X, Wang X and Dougherty ER (2006). Multi-class cancer classification using multinomial probit regression with Bayesian gene selection. Syst. Biol. 153: 70-78. 